\title{
A critical pathway reduced resource use without compromising safety and effectiveness in community acquired pneumonia
}

\author{
Marrie TJ, Lau CY, Wheeler SL, et al., for the CAPITAL Study Investigators. A controlled trial of a critical pathway for \\ treatment of community-acquired pneumonia. JAMA $2000 \mathrm{Feb}$ 9;283:749-55.
}

QUESTION: In patients with community acquired pneumonia (CAP), does a critical pathway reduce the use of resources without compromising safety and effectiveness?

Design

Randomised (cluster randomisation), blinded (outcome assessor), ${ }^{*}$ controlled trial with 6 week follow up.

Setting

20 teaching or community hospitals in Canada.

Patients

1743 patients who had $\geqslant 2$ signs or symptoms of CAP (eg, temperature $>38^{\circ} \mathrm{C}$, productive cough, chest pain, shortness of breath, and crackles on auscultation) and opaque findings on chest radiography that were consistent with the presence of acute pneumonia. Exclusion criteria included HIV infection; use of prednisone, $>10 \mathrm{mg} /$ day, or other immunosuppressive agents; treatment for cancer; history of organ transplantation; active tuberculosis; or cystic fibrosis. Patients in 19 of 20 hospitals were evaluated.

\section{Intervention}

After stratification by type of institution, hospitals were matched by historical length of stay and allocated to a critical pathway $(n=10)$ or to conventional management $(n=10)$. The critical pathway had 3 components: a clinical prediction rule for admission decisions, levofloxacin treatment, and practice guidelines for the care of inpatients. Levofloxacin was given to admitted patients, $500 \mathrm{mg} /$ day intravenously, and to patients who were sent home, $500 \mathrm{mg}$ /day orally for 10 days. The practice guidelines included criteria for discharge.

Janssen-Ortho Inc and

Medical Research

Council of

Canada-Pharmaceutical

Manufacturers'

Association of Canada

Awards Program.

For correspondence:

Dr B G Feagan,

London Clinical Trials

Research Group, John P

Robarts Research

Institute, 100 Perth

Drive, London, Ontario

N6A 5 K8, Canada.

Fax +1519663

3807 .

\section{Main outcome measures}

The main efficacy outcome was the score on the ShortForm 36 Physical Component Summary (SF-36 PCS) scale. Resource use was assessed by the number of bed rest days per patient managed, which was the product of the mean length of stay and admission rate.

\section{Main results}

The groups did not differ in SF-36 PCS scores. Patients in the critical pathway group had fewer bed rest days per patient managed $(\mathrm{p}<0.04)$ than did those in the conventional management group (table).

A critical pathway $(C P)$ v conventional management (control) in community acquired pneumoniat

\begin{tabular}{llll}
\hline Outcome at 6 weeks & CP & Control & $\begin{array}{l}\text { Mean difference } \\
(95 \% \mathrm{Cl})\end{array}$ \\
\hline Bed rest days per managed patient & 4.4 & 6.1 & $1.7(1.5$ to 1.9) \\
\hline
\end{tabular}

tMean difference and $\mathrm{Cl}$ calculated from data in article.

\section{Conclusions}

In patients with community acquired pneumonia, a critical pathway used fewer resources than did conventional management, and the 2 methods were similarly effective and safe.

*See glossary.

\section{COMMENTARY}

This groundbreaking study by Marrie et al found that a critical pathway for CAP could maintain quality while decreasing resource use. The 2 groups of hospitals differed in the median but not the mean length of stay. Use of the median rather than the mean makes sense for a probably skewed distribution. If each hospital day costs the same, however, the mean length of stay might best reflect the overall hospital cost. The authors are conducting a formal cost analysis to investigate this issue.

Because levofloxacin was provided only for hospitals in the critical pathway group, chart review to assess the number of antibiotics prescribed was probably biased by knowledge of hospital status.

Lack of blinding may have contributed to admission of generally healthier patients to the intervention group of hospitals. Knowing that the critical pathway hospitals used the severity of illness scores for admission, the nurse(s) might have been biased to give inappropriately high scores to these patients. Admitting healthier patients would have achieved all of the critical pathway group outcomes: reduced median length of stay, fewer antibiotics per patient, fewer days of intravenous antibiotic administration, and sicker patients. The outcome called bed rest days per patient managed addressed this bias by assessing both inpatient care (length of stay) and admission policy (admission rate). The finding of fewer bed rest days per patient managed in the critical pathway group argues against any bias toward scoring patients as "sicker" and thereby admitting healthier patients. Future research could address which critical pathway features contributed most to efficient care. Is the most efficient intervention simply using the drug levofloxacin, or is it using a protocol for the different decision points of admission, for switching to oral antibiotics, and for discharge?

Do the results from Canadian hospitals apply to other hospitals? The average length of stay for US hospitals in 1996 was shorter than that reported in this study, and reimbursement systems differ between the 2 countries. Application of this and similar critical pathways to representative groups of hospitals will be an exciting area for future study. Alec Chessman, MD Medical University of South Carolina Charleston, South Carolina, USA 